\title{
HARMONIC ANALYSIS AND CENTERS OF GROUP ALGEBRAS
}

BY

\author{
J. LIUKKONEN( $\left.{ }^{1}\right)$ AND R. MOSAK (2)
}

\begin{abstract}
The purpose of this paper is to present some results of harmonic analysis on the center of the group algebra $Z\left(L^{1}(G)\right)$ where $G$ is a locally compact group. We prove that $Z\left(L^{1}(G)\right)$ is a regular, Tauberian, symmetric Banach *algebra and contains a bounded approximate identity. Wiener's generalized Tauberian theorem is therefore applicable to $Z\left(L^{1}(G)\right)$. These results complement those of $I$. E. Segal relating to the group algebra of locally compact abelian and compact groups. We also prove that if $G$ contains a compact normal subgroup $K$ such that $G / K$ is abelian, then $Z\left(L^{1}(G)\right)$ satisfies the condition of Wiener-Ditkin, so that any closed set in its maximal ideal space whose boundary contains no perfect subset is a set of spectral synthesis. We give an example of a general locally compact group for which $Z\left(L^{1}(G)\right)$ does not satisfy the condition of Wiener-Ditkin.
\end{abstract}

Introduction. The purpose of this paper is to present some results of harmonic analysis on the center of the group algebra $Z\left(L^{1}(G)\right)$ where $G$ is a locally compact group. We prove that $Z\left(L^{1}(G)\right)$ is a regular, Tauberian, symmetric Banach *-algebra containing a bounded approximate identity. Wiener's generalized Tauberian theorem is therefore applicable to $Z\left(L^{1}(G)\right)$ : If $M$ is a closed proper ideal in $Z\left(L^{1}(G)\right)$, then $M$ is contained in a regular maximal ideal. These results complement those of Segal [17] relating to the group algebra of locally compact abelian and compact groups.

Our results depend strongly upon the theory of groups with "compactness conditions" [4]. The most immediate reason for this (see [11]) is that $Z\left(L^{1}(G)\right.$ ) is nontrivial iff $G$ is an $[I N]$ group ( $G$ contains a compact neighborhood of the identity, invariant under inner automorphisms). Moreover, we show that, roughly speaking, whenever $G$ is an $[I N]$ group, there is a group $G_{1}$ satisfying a much stronger compactness condition (an $[F I A]_{B}^{-}$group) such that $Z\left(L^{1}(G)\right)$ is isomorphic as a Banach *-algebra to a very well-behaved subalgebra of $Z\left(L^{1}\left(G_{1}\right)\right)$. We are therefore able to restrict our attention largely to groups satisfying strong compactness conditions, for which some structure theory is available [4].

Received by the editors July 21, 1972 and, in revised form, July 3, 1973.

AMS (MOS) subject classifications (1970). Primary 22D15.

Key words and phrases. Centers of group algebras, regular algebras, Tauberian algebras, symmetric *-algebras, approximate identities, condition of Wiener-Ditkin, [IN] groups, $[F C]^{-}$groups, $[F I A]^{-}$groups, spherical functions.

(1) Research partially supported by National Science Foundation Grant NSF-GP 28655.

(2) Research partially supported by National Science Foundation Grant NSF-GP 30673.

Copyright $\bigcirc 1974$, American Mathematical Society 
We recall briefly from [4] the classes of locally compact groups that we shall need; here $G$ is locally compact and $B$ is a subgroup of Aut $(G)$, the group of bicontinuous automorphisms of $G$.

$[I N]_{B}: G$ contains a compact $B$-invariant neighborhood of the identity.

$[S I N]_{B}: G$ contains a fundamental system of $B$-invariant neighborhoods at the identity.

$[F D]_{B}^{-}:$The $B$-commutators $\left\{\beta(x) x^{-1}: \beta \in B, x \in G\right\}$ generate a relatively compact subgroup of $G$.

$[F C]_{B}^{-}$: Every $B$-orbit $B(x)=\{\beta(x): \beta \in B\}$ is relatively compact.

$[F I A]_{B}^{-}: B$ is relatively compact in $\operatorname{Aut}(G)$.

When $B=I(G)$ (the inner automorphisms of $G$ ) we omit the subscript $B$; we shall in general assume $B \supset I(G)$. We recall also that $G$ is an $[F I A]_{B}^{-}$group iff $G \in[F C]_{B}^{-} \cap[S I N]_{B}$.

The organization of the paper is as follows. In $\$ 1$ we state the main results concerning $Z\left(L^{1}(G)\right)$ for general locally compact groups and prove several propositions reducing its study to the case $G \in[F I A]_{B}^{-}$. $\$ 2$ is devoted to a proof that $Z\left(L^{1}(G)\right)$ is regular and Tauberian. Finally, in $\$ 3$, we show that the algebra $Z\left(L^{1}(G)\right)$ satisfies the condition of Wiener-Ditkin in case $G \in[F D]^{-}$; we also give an example to show that the condition does not hold for general $G$.

After this paper was prepared for typing, we received a preprint entitled $O n$ complete regularity of group algebras, by $\mathrm{E}$. Kaniuth and D. Steiner. In their paper they prove our Propositions 2.4 and 2.6, as well as Corollary 2.5. Their method of proof, however, is quite different from ours; they introduce a convolution for functions on $X(G)$ and study the Banach algebra $L^{1}(\mathscr{X}(G)$ ).

1. Let $G$ be a locally compact group, and $B$ be a subgroup of Aut $(G)$ with $B \supset I(G)$ where $I(G)$ is the group of inner automorphisms. Suppose that the elements of $B$ are Haar-measure-preserving (this will be valid in all the cases we study in the paper). Then we define the $B$-center of $L^{1}(G), Z^{B}\left(L^{1}(G)\right)$, to be the set of all $f \in L^{1}(G)$ such that $f^{\beta}=f$ for all $\beta \in B$ (where $f^{\beta}(x)=f\left(\beta^{-1} x\right)$ ). $Z^{B}\left(L^{1}(G)\right)$ is a commutative Banach *-subalgebra of $L^{1}(G)$, and is even central, since the center $Z\left(L^{1}(G)\right)$ of $L^{1}(G)$ is exactly $Z^{l(G)}\left(L^{1}(G)\right)$. Also, $Z^{B}\left(L^{1}(G)\right.$ ) is semisimple, since it admits a faithful *-representation on $L^{2}(G)$.

We recall for future use some facts about $B$-characters of $G$, when $B \supset I(G)$. If $\varphi$ is a continuous positive-definite function on $G$, then $\varphi$ defines a strongly continuous unitary representation $\pi$ of $G$ on a Hilbert space $\mathcal{H}$, with a cyclic vector $u \in \mathcal{H}$ such that $\varphi(x)=\langle\pi(x) u, u\rangle$ for all $x \in G$. We shall say that $\varphi$ defines the cyclic representation $(\mathcal{H}, \pi, u)$. If $\varphi$ is $B$-invariant $\left(\varphi^{\beta}=\varphi\right.$ for all $\beta \in B$ ) then one can define a continuous unitary representation $\beta \mapsto U(\beta)$ of $B$ on $\mathcal{H}$ such that $U(\beta)_{\pi}(x) U(\beta)^{-1}=\pi(\beta x)$ for $x \in G, \beta \in B$; moreover $u$ is $B$. 
fixed $(U(\beta) u=u$ for all $\beta)[10, \S 2]$. If $\varphi$ is $B$-invariant and $\varphi(1)=1$, one can show $[10,2.7]$ that the space of $B$-fixed vectors is one-dimensional iff $\varphi$ is an extreme point of the set of all continuous positive definite $B$-invariant functions of norm $\leq 1$. In this case we say $\varphi$ is a $B$-character, and write $\mathcal{X}^{B}(G)$ for the set of $B$-characters (we refer simply to characters, and write $\mathcal{X}(G)$, in case $B=I(G)$ ). Any $\varphi \in X^{B}(G)$ defines a multiplicative linear functional $\Phi$ on $Z^{B}\left(L^{1}(G)\right): \Phi(f)=\hat{f}(\varphi)=\int_{G} f(x) \varphi(x) d x$. Every $B$-character is normalized $(\varphi(1)=1)$ so the (Hausdorff) weak-* topology that $X^{B}(G)$ inherits as a subspace of $L^{\infty}(G)$ coincides with the topology of uniform convergence on compacta of $G[1,13.5 .2]$.

Suppose now that $G$ is an $[F I A]_{B}^{-}$group, with $B \supset I(G)$. In this case one can give other useful descriptions of $B$-characters.

1.1. Proposition. Let $G$ be an $[F I A]_{B}^{-}$group, with $B \supset I(G)$. If $\varphi$ is a continuous nonzero positive-definite $B$-invariant function on $G$, then the following are equivalent:

(i) $\varphi$ is a B-character;

(ii) $\Phi$ is a multiplicative linear functional on $Z^{B}\left(L^{1}(G)\right)$;

(iii) $\varphi$ satisfies the B-character formula

$$
\phi(x) \phi(y)=\int_{B^{-}} \phi(x \cdot \beta(y)) d \beta
$$

( $d \beta$ is normalized Haar measure on the compact group $B^{-}$).

Moreover, the map $\varphi \mapsto \Phi$ is a bomeomorphism of $X^{B}(G)$ onto the maximal ideal space of $Z^{B}\left(L^{1}(G)\right)$. The one-point compactification of $X^{B}(G)$ can be identified with $X^{B}(G) \cup\{0\}$ in the weak.* topology.

Proof. The first assertion is contained in Proposition 4.4 of [10]. The second follows from results of Hulanicki [7] (see also [10, Proposition 4.12]). The last assertion is Corollary 4.2 of [10].

We shall show next that the study of $Z\left(L^{1}(G)\right), G$ a locally compact group, may be reduced to the study of $Z^{B}\left(L^{1}(H)\right)$, for an appropriate $[F I A]_{B}^{-}$group $H$. We first assume that $G \in[I N]_{B}$, with $B \supset I(G)$. Let $K$ be the intersection of all compact $B$-invariant neighborhoods of 1 . It follows from the proof of Theorem (2.5) of [4] that $K$ is a compact $B$-invariant subgroup of $G$ (in particular $K$ is normal). Let $B^{\prime}$ denote the group of automorphisms of $G^{\prime}=G / K$ induced by $B$; then $B^{\prime} \supset I\left(G^{\prime}\right)$. Let $\theta$ denote the canonical projection of $G$ onto $G^{\prime}$.

1.2. Proposition. With the above hypotheses and notation, $G^{\prime}=G / K$ is an $[S I N]_{B}$ group, and the map $g \mapsto g \circ \theta$ is an isometric *-isomorphism of the Banacb algebra $Z^{B}{ }^{\prime}\left(L^{1}\left(G^{\prime}\right)\right)$ onto $Z^{B}\left(L^{1}(G)\right)$. Moreover, $\varphi \mapsto \varphi \circ \theta$ is a bomeomorpbism of $X^{B^{\prime}}\left(G^{\prime}\right)$ onto $X^{B}(G)$. 
Proof. Theorem (2.5) of [4] implies that $G / K$ is an $[S I N]_{B}$ group. To prove the second assertion, we recall some facts from Theorem (28.55) of [6]. Following [6], we denote by $\mathcal{F}$ the set of all $f \in L^{1}(G)$ such that $f$ agrees almost everywhere with an $L^{1}$ function which is constant on cosets of $K$. It follows from (28.55) of [6] and its proof that the map $g \mapsto g \circ \theta$ is an isometric *-algebra isomorphism of $L^{1}(G / K)$ onto $\mathcal{F} \subset L^{1}(G)$ and that the inverse is the map $f \mapsto f^{\prime}$, where $f^{\prime}(\dot{x})=\int_{K} f(x k) d k$ ( $d k$ is normalized Haar measure on $K$ ). To prove the second assertion, therefore, we must verify: (i) $Z^{B}\left(L^{1}(G)\right)$ is contained in $\mathcal{F}$; (ii) if $f \in Z^{B}\left(L^{1}(G)\right)$, then $f^{\prime} \in Z^{B^{\prime}}\left(L^{1}\left(G^{\prime}\right)\right)$; (iii) if $g \in Z^{B^{\prime}}\left(L^{1}\left(G^{\prime}\right)\right)$, then $g \circ \theta$ $\epsilon Z^{B}\left(L^{1}(G)\right.$ ). For (i), we show first that if $\varphi$ is any continuous $B$-invariant function on $G$, and $k \in K$, then $\varphi(k)=\varphi(1)$. But if $V$ is a fixed compact $B$-invariant neighborhood of 1 in $G$ and $\epsilon>0$ is arbitrary, then $V \cap\{t \in G:|\varphi(t)-\varphi(1)|$ $\leq \epsilon\}$ is a compact $B$-invariant neighborhood of 1 ; hence by the definition of $K,|\varphi(k)-\varphi(1)| \leq \epsilon$ for all $k \epsilon K$. Since $\epsilon>0$ is arbitrary, $\varphi(k)=\varphi(1)$. Now choose $f \in Z^{B}\left(L^{1}(G)\right)$, and set $b=f /|f|^{1 / 2}(b(x)=0$ when $f(x)=0)$; then $b$ is a $B$-invariant $L^{2}$ function. Let $\rho$ denote the right regular representation of $G$ on $L^{2}(G)$ (or $\left.L^{1}(G)\right)$, and let $\varphi(x)=b^{2} * h(x)=\left\langle p(x) h, b{ }_{L^{2}(G)}\right.$ (here $b^{2}(x)=$

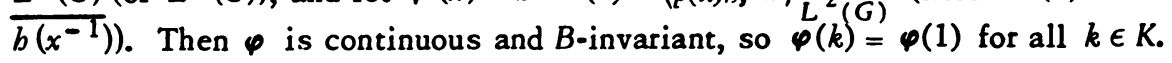
The limiting case of the Schwarz inequality then implies that $\rho(k) b=b$ for all $k \in K$; it follows easily that $\rho(k) f=f$ for all $k \in K$. We conclude from (28.68) (iii) of [6] that $f$ is equal a.e. to a function constant on $K$ cosets, that is, $f \in \mathcal{F}$. This completes the proof of (i), while the proofs of (ii) and (iii) are clear; hence the second assertion is proved.

For the last assertion, let $\varphi$ be a continuous positive definite $B$-invariant function on $G$; then for $x \in G$ and $k \in K$,

$$
|\varphi(x k)-\varphi(x)|^{2} \leq 2 \varphi(1)(\varphi(1)-\operatorname{Re} \varphi(k))=0
$$

Hence $\varphi$ is constant on $K$ cosets. Thus the map $\varphi \mapsto \varphi \circ \theta$, which clearly preserves extremal properties, is a bijection (and a homeomorphism) of $X^{B^{\prime}}\left(G^{\prime}\right)$ onto $\mathfrak{X}^{B}(G)$.

We remark that one can show, using [10, Corollary 3.4], that $K$ is exactly the set of $x \in G$ such that $\varphi(x)=1$ for all $\varphi \in X^{B}(G)$ (compare also [8, p. 336]). Thus for all practical purposes, the theory of $B$-characters of $[I N]_{B}$ groups $(B)$ $I(G))$ can be reduced to the theory of $B$-characters of $[S I N]_{B}$ groups. The following easy corollary is stated in Hulanicki [7] for the case $B=I(G)$.

1.3. Corollary. Let $G$ be an $[F C]_{B}^{-}$group, with $B \supset I(G)$. Then the map $\varphi \mapsto \Phi$ is a bomeomorphism of $\mathscr{X}^{B}(G)$ onto the maximal ideal space of $Z^{B}\left(L^{1}(G)\right)$ (recall that $\Phi(f)=\hat{f}(\varphi)$ for $f$ in $Z^{B}\left(L^{1}(G)\right)$ ). 
Proof. $G$ is an $[I N]_{B}$ group (see Theorem 2.2 of [9], as well as [16]); hence 1.2 applies. Since the maximal ideal space of $Z^{B}{ }^{\prime}\left(L^{1}\left(G^{\prime}\right)\right)$ can be identified with $X^{B^{\prime}}\left(G^{\prime}\right)$ (Proposition 1.1), and the map $f \mapsto f^{\prime}$ is an isomorphism of $Z^{B}\left(L^{1}(G)\right)$ onto $Z^{B^{\prime}}\left(L^{1}\left(G^{\prime}\right)\right)$, it suffices to prove that $\hat{f}(\varphi \circ \theta)=\hat{f}^{\prime}(\varphi)$, for $f \epsilon$ $Z^{B}\left(L^{1}(G)\right)$ and $\varphi \in X^{B^{\prime}}\left(G^{\prime}\right)$. But this is a simple computation.

We now suppose that $G$ is an $[I N]$ group. Let $G_{0}$ be the set of all $x \in G$ such that the conjugacy class $\left\{y x y^{-1}: y \in G\right\}$ is relatively compact. $G_{0}$ is clearly a normal subgroup, and since $G$ contains a compact $I(G)$-invariant neighborhood of $1, G_{0}$ is open. Let $B$ denote the restriction to $G_{0}$ of the inner automorphisms of $G$. Then $B \supset I\left(G_{0}\right)$, and $G_{0}$ is an $[F C]_{B}^{-}$group. For $f \in L^{1}(G)$, let $f_{0}$ denote the restriction of $f$ to $G_{0}$ (clearly $f_{0} \in L^{1}\left(G_{0}\right)$ ).

1.4. Proposition. Let $G$ be an $[I N]$ group. With notation as above, the map $f \mapsto f_{0}$ is an isometric *algebra isomorphism of $Z\left(L^{1}(G)\right)$ onto $Z^{B}\left(L^{1}\left(G_{0}\right)\right)$. Also, the map $\varphi \mapsto \Phi$ is a continuous surjection of $\mathcal{X}(G)$ onto the maximal iaeal space of $Z\left(L^{\mathrm{r}}(G)\right)$.

Proof. By Lemma 2 of [12], every $f \in Z\left(L^{1}(G)\right)$ vanishes almost everywhere off $G_{0}$. Therefore $f \mapsto f_{0}$ is clearly an isomorphism. Since the maximal ideal space of $Z^{B}\left(L^{1}\left(G_{0}\right)\right)$ is identified with $X^{B}\left(G_{0}\right)$ (Corollary 1.3), the second assertion follows immediately from the fact that $\varphi \mapsto \varphi_{0}$ is a continuous surjection of $X(G)$ onto $X^{B}\left(G_{0}\right)$ [10, Proposition 2.9].

1.5. Corollary. Let $G$ be a locally compact group. If $Z\left(L^{1}(G)\right) \neq(0)$, then there exists an $[F I A]_{B}^{-}$group $H$, with $B \supset I(H)$, such that $Z\left(L^{1}(G)\right)$ is isomorphic as a Banach *algebra to $Z^{B}\left(L^{1}(H)\right)$.

Proof. The hypothesis implies that $G$ is an [IN] group [11]. Hence the conclusion follows from 1.4 and 1.2 , where 1.2 is applied to the $[F C]_{B}^{-}$group $G_{0}$.

Recall that a commutative Banach *-algebra is symmetric if each multiplicative linear functional is hermitian.

1.6. Corollary. Let $G$ be a locally compact group. Then $Z\left(L^{1}(G)\right)$ is a symmetric Banach *algebra with bounded approximate identity.

Proof. The first assertion follows from Corollary 1.5, together with the description (1.1) of the maximal ideal space of $Z^{B}\left(L^{1}(G)\right)$, where $G \in[F I A]_{B}^{-}$(B) $I(G)$ ). For the second assertion we remark that when $G \in[S I N]_{B}$, the (suitably normalized) characteristic functions of compact $B$-invariant neighborhoods of 1 form a bounded approximate identity for $L^{1}(G)$, hence a fortiori for $Z^{B}\left(L^{1}(G)\right.$ ).

The following corollary was proved for ableian groups by Porcelli and Collins [13]. 
1.7. Corollary. Let $G$ be a locally compact group. Every maximal ideal in $Z\left(L^{1}(G)\right)$ is regular and therefore closed.

Proof. Since $Z\left(L^{1}(G)\right)$ contains a bounded approximate unit, it follows from Cohen's factorization theorem $[6,32.26]$ that $Z\left(L^{1}(G)\right) * Z\left(L^{1}(G)\right)=Z\left(L^{1}(G)\right)$. The result now follows as in [13].

We now state some other results about $Z\left(L^{1}(G)\right)$, for locally compact $G$; the proof of 1.8 , which uses Corollary 1.5 in the reduction to the case of $[F I A]_{B}^{-}$ groups, will be given in $\$ 2$. Recall that a commutative Banach algebra is regular if its Gel'fand transforms separate points from closed sets (equivalently, compact sets from closed sets) in the maximal ideal space. We say that a commutative Banach algebra is Tauberian [15] (a Wiener algebra in the terminology of [14]) if the elements whose Gel'fand transforms have compact support are dense in the algebra. Analogues of the next two results are proved in Willcox [18] in the case that $G$ is a direct product of an abelian and a compact group.

1.8. Theorem. Let $G$ be a locally compact group. Then $Z\left(L^{1}(G)\right)$ is a regular Tauberian Banacb algebra.

Analogues of 1.9 and 1.10 were proved in [3] for central topological groups.

1.9. Corollary (Wiener's Tauberian theorem). Let $M$ be a closed proper ideal in $Z\left(L^{1}(G)\right.$ ). Then $M$ is contained in a regular maximal ideal.

Proof. Since $Z\left(L^{1}(G)\right)$ is semisimple, this follows from 1.8 together with, for example, Theorem 39.27 of [6].

The hypothesis of the next corollary implies that $G \in[I N]$, so that we may use the results and notation of 1.2.

1.10. Corollary. Let $f \in Z\left(L^{1}(G)\right)$, and suppose $\hat{f}$ does not vanish anywhere in $\mathcal{X}(G)$. Then any element of $\mathcal{F}$ can be approximated in $L^{1}$ by linear combina. tions of translates of $f$.

Proof. By 1.2 we may assume that $G$ is actually an $[S I N]$ group, and $\mathcal{F}=$ $L^{1}(G)$. By 1.4 the Gel'fand transform of $f$ vanishes at no point of the maximal ideal space of $Z\left(L^{1}(G)\right)$; hence by 1.9 the ideal generated by $f$ in $Z\left(L^{1}(G)\right.$ ) is all of $Z\left(L^{1}(G)\right)$. If $T$ denotes the closed linear span in $L^{1}$ of the set of translates of $f$, then $T$ is a closed ideal in $L^{1}$ whose intersection with $Z\left(L^{1}(G)\right)$ is all of $Z\left(L^{1}(G)\right)$. Since $Z\left(L^{1}(G)\right)$ contains an approximate identity for $L^{1}(G), T$ must be all of $L^{1}(G)$.

2. In this section we present the proof of Theorem 1.8. The proof proceeds by a series of reductions to the case of $[F D]^{-}$groups, and we therefore need some detailed information about characters of such groups. It is convenient to use the 
theory of spherical functions to present this information, and we briefly recall here some facts from [2] and [5] about spherical functions. Our terminology and notation differ slightly from that in [5].

Let $G$ be a locally compact unimodular group, and $B$ a compact subgroup of $G$. A function $f$ on $G$ is said to be bi-invariant (with respect to $B$ ) if for each $b_{1}, b_{2} \in B, f\left(b_{1} x b_{2}\right)=f(x)$ for all $x \in G$ (if $f$ is an $L^{1}$ function, the last quantifier should read "for almost all $x \in G$ "). Suppose $\varphi$ is a continuous positive-definite function on $G$, defining the cyclic representation $(\mathcal{H}, \pi, u)$. It is easy to see that $u$ is a $B$-fixed vector $(\pi(b) u=u$ for all $b \in B)$ if and only if $\varphi$ is bi-invariant. Hence if we denote by $\mathcal{H}^{b}$ the space of $B$-fixed vectors, then $\mathcal{H}^{\natural} \neq(0)$ iff some continuous positive-definite function associated with $\pi$ is bi-invariant. In this case we say that $\pi$ is of class 1 . Let $L^{4}(G)$ denote the *-subalgebra of $L^{1}(G)$ consisting of bi-invariant functions. Suppose $L^{\natural}(G)$ is commutative, and let $\varphi$ be a continuous, nonzero, bounded, bi-invariant function on $G$. Then $\varphi$ is a spherical function (with respect to $B$ ) if the map $f \mapsto$ $\int_{G} f(x) \varphi(x) d x$ is a multiplicative linear functional on $L^{q}(G)$.

2.1. Proposition. With the above notation, suppose $L^{b}(G)$ is commutative, and let $\varphi$ be a continuous positive-definite bi-invariant function on $G$ with $\varphi(1)=1$. Then the following are equivalent:

(i) $\pi$ is irreducible;

(ii) $\mathcal{H}^{\natural}$ is one dimensional;

(iii) $\varphi$ is a spherical function.

Moreover, the map $\varphi \mapsto \pi$ is a bijection from the set of positive-definite spberical functions onto the set of equivalence classes of irreducible unitary representations of class 1 .

Proof. See [2] and [5, Chapter 10].

2.2. Proposition. Let $G$ be a locally compact unimodular group, $K$ a compact normal subgroup of $G, B$ a compact subgroup of $K$. Suppose that $L^{\natural}(G)$ is commutative, and let $\varphi$ be a positive-definite spberical function on $G$ (with respect to $B)$. There is an open subgroup $S=S(\varphi)$ containing $K$ with the follow. ing properties:

(i) $S(\varphi)=\{x \in G: \varphi(y) \neq 0$ for some $y \in x K\}$.

(ii) The function $x \mapsto \int_{K}|\varphi(x k)|^{2} d k$ is constant and nonzero on $S$ and zero on $G \backslash S$. (Here dk is normalized Haar measure on $K_{.}$)

If $\lambda$ is an ordinary one-dimensional character of $S$, constant on cosets of $K$ (briefly, $\left.\lambda \in(S / K)^{\wedge}\right)$ let $\lambda^{\sim}$ denote the trivial extension of $\lambda$ to $G\left(\lambda^{\sim}(x)=0\right.$ for $x \in G \backslash S$ ); also suppose that $\psi$ is another spberical function on $G$ with re. spect to $B$. 
(iii) If the restrictions $\varphi_{K}$ and $\psi_{K}$ to $K$ are equal, then there exists a unique $\lambda \epsilon(S / K)^{\wedge}$ such that $\psi=\lambda^{\sim} \bullet$.

(iv) If $\varphi_{K} \neq \psi_{K^{\prime}}$ then $\int_{K} \varphi(x k) \overline{\psi(x k)} d k=0$ for all $x \in G$.

Proof. Let $(\mathcal{H}, \pi, u)$ be the irreducible unitary representation of $G$ defined by $\varphi$, and let $\mathcal{H}_{1}$ be the closed linear span of $\{\pi(k) u: k \in K\}$. For each $k \in K$, let $\pi_{1}(k)$ denote the restriction to $H_{1}$ of $\pi(k)$; then $\pi_{1}$ is a continuous unitary representation of $K$ on $\mathcal{H}_{1}$. Since $\mathcal{H}^{\xi}$, the space of $B$-fixed vectors, is one-dimensional and generated by $u \in \mathcal{H}_{1},\left(\mathcal{H}_{1}\right)^{4}$ is also one-dimensional. Since the cyclic unitary representation of $K$ defined by $\varphi_{K}$ is, up to equivalence, $\left(\mathcal{H}_{1}\right.$, $\left.\pi_{1}, u\right)$, it follows from 2.1 that $\varphi_{K}$ is a spherical function, $\pi_{1}$ is irreducible, and therefore $\mathcal{H}_{1}$ is finite-dimensional. Moreover, any other component of the restriction $\pi_{K}$ of $\pi$ to $K$ which is equivalent to $\pi_{1}$ must also contain a $B$-fixed nonzero vector, orthogonal to $u$, which is impossible; hence the multiplicity of $\pi_{1}$ in $\pi_{K}$ must be one. Let $S=S(\varphi)$ be the stability group of $\pi_{1}$ in $G$, under the (continuous) action of $G$ on $\hat{K}$ by conjugation; since $\hat{K}$ is discrete, $S$ is open in $G$. Then $\pi_{K}$ is the orthogonal direct sum of the conjugates $\left(\pi_{1}\right)^{x_{i}}$, where $x_{i}$ runs through a complete set of representatives for the coset space $G / S$. Moreover $\pi_{1}$ is the only representation in the orbit $\left\{\left(\pi_{1}\right)^{x_{i}}\right\}$ which is of class 1 .

Now let $\mathcal{H}_{2}$ be the closed linear span in $\mathcal{H}$ of the set $\{\pi(x) u: x \in S\}$, and for $x \in S$, let $\pi_{2}(x)$ be the operator $\pi(x)$ restricted to $\mathcal{H}_{2}$. As above $\pi_{2}$ is a continuous irreducible unitary representation of $S$ on $\mathcal{H}_{2} ; \pi_{2}$ is of class 1 (since $u \in \mathcal{H}_{2}$ ), and $\mathcal{H}_{1} \subset \mathcal{H}_{2}$. But the same analysis as above, with $S$ replacing $G$, shows that $\left(\pi_{2}\right)_{K}$ is the direct sum of all the inequivalent conjugates of $\pi_{1}$ under the action of $S$ on $\hat{K}$. Since $S$ stabilizes $\pi_{1},\left(\pi_{2}\right)_{K}=\pi_{1}$; that is, $\mathcal{H}_{2}=\mathcal{H}_{1}$. Thus for each $x \in S, \mathcal{H}_{1}$ is invariant under $\pi(x)$. On the other hand, for $x \in G \backslash S$, $\pi(x) \mathcal{H}_{1}$ is orthogonal to $\mathcal{H}_{1}$. In fact, normality of $K$ shows that $\pi(x) \mathcal{H}_{1}$ is invariant under the operators $\pi(k)(k \in K)$, and an immediate calculation shows that $\pi(x)$ is an intertwining operator for this representation of $K$ on $\pi(x) \mathcal{H}_{1}$ and the representation $\left(\pi_{1}\right)^{x^{-1}}$. of $K$ on $\mathcal{H}_{1}$. Therefore $\pi(x) \mathcal{H}_{1}$ is the unique subspace of $\mathcal{H}$ on which $\pi(k)$ acts as $\left(\pi_{1}\right)^{x^{-1}}(k)$ for each $k \in K$, so $\pi(x) \mathcal{H}_{1}$ is orthogonal to $\mathcal{H}_{1}$. Thus for $k \in K$ and $x \in G \backslash S, \varphi(x k)=\left\langle\pi(k) u, \pi\left(x^{-1}\right) u\right\rangle=0$, while for $x \in S, k \mapsto \varphi(x k)=\left\langle\pi_{1}(k) u, v\right\rangle$ (with $v=\pi\left(x^{-1}\right) u \in \mathcal{H}_{1}$, since $x \in S$ ) is a coordinate function associated with $\pi_{1}$. Now (ii) follows immediately from the orthogonality relations for compact groups, and (i) follows from (ii) and the continuity of $\varphi$.

For (iv), if $\psi$ is another positive-definite spherical function on $G$ and $\psi_{K} \neq$ $\varphi_{K}$, then $\psi_{K}$ and $\varphi_{K}$ are distinct positive-definite spherical functions on $K$ and hence (by 2.1) define inequivalent representations $\pi_{1}$ and, say, $\sigma_{1}$, of $K$. 
Therefore $\int_{K} \varphi(x k) \overline{\psi(x k)} d k=0$ unless $x$ is in $S(\varphi) \cap S(\psi)$, that is, stabilizes both $\pi_{1}$ and $\sigma_{1}$, and if $x$ stabilizes both $\pi_{1}$ and $\sigma_{1}$ then the integral is the inner product of coordinate functions associated with inequivalent representations of $K$. Assertion (iv) then also follows from the orthogonality relations.

To prove (iii) we observe first that the previous arguments show that if $\varphi_{K}=$ $\psi_{K}$ then $S(\varphi)=S(\psi)=S$. Moreover the arguments above also show that in this case we may take irreducible unitary representations $\pi$ and $\sigma$ of $S$ on a finitedimensional Hilbert space $\mathcal{H}_{1}$ and a vector $u \in \mathcal{H}_{1}$ such that: $\pi_{K}=\sigma_{K}=\pi_{1}$ is an irreducible representation of $K$ on $\mathcal{H}_{1} ; \varphi(x)=\langle\pi(x) u, u\rangle$ and $\psi(x)=\langle\sigma(x) u, u\rangle$ for all $x \in S$. Now for all $x \in S$ and $k \in K$ we have

$$
\pi(x)^{-1} \pi_{1}(k) \pi(x)=\pi_{1}\left(x^{-1} k x\right)=\sigma(x)^{-1} \pi_{1}(k) \sigma(x) .
$$

It follows that each $\pi(x) \sigma(x)^{-1}$ is an intertwining operator for the irreducible representation $\pi_{1}$ and hence, by Schur's lemma, $\pi(x)=\lambda(x) \sigma(x)$ for a unique scalar $\lambda(x)$ of absolute value 1 . Clearly we have $\lambda(x y)=\lambda(x) \lambda(y)$ and $\lambda(k)=$ 1 for all $x, y \in S$ and $k \in K$. Also $\varphi(x)=\lambda(x) \psi(x)$ for all $x \in S$. It follows that $\lambda$ is continuous at 1 ; hence $\lambda \in(S / K)$. The uniqueness of $\lambda$ follows from the description of $S$ in (i).

We now turn to the case of an $[F I A]_{B}^{-}$group $G$, where $B \supset I(G)$. Let $G^{*}$ be the semidirect product $G^{*}=G \times{ }_{\eta} B^{-}$(where the compact group $B^{-} C$ Aut $(G)$ acts in the natural way), and let $B^{*}=\{1\} \times B^{-}$. As was observed in $[10, \$ 4]$, there is a close connection between the theory of spherical functions on $G^{*}$ (with respect to $B^{*}$ ) and the theory of $B$-characters on $G$. In fact, it is easy to see that the map $f \mapsto f^{*}$, where $f^{*}(x, \beta)=f(x)$, is an isometric *-algebra isomorphism of $Z^{B}\left(L^{1}(G)\right)$ onto $L^{*}\left(G^{*}\right)$; in particular, this latter algebra is commutative. If $\varphi$ is a $B$-character of $G$, defining the cyclic unitary representation $(\mathcal{H}, \pi, u)$ of $G$, and the representation $U$ of $B^{-}$, we set $\pi^{*}(x, \beta)=\pi(x) U(\beta)$. Then $\pi^{*}$ is a continuous unitary representation of $G^{*}$, of class 1 with respect to $B^{*}$, and $\mathcal{H}^{\natural}$ is one-dimensional. Therefore the function $\varphi^{*}$ on $G^{*}, \varphi^{*}(x, \beta)=$ $\varphi(x)$, is a positive-definite spherical function on $G^{*}$; moreover, all positive definite spherical functions on $G^{*}$ are obtained in this way from a (unique) $B$ character of $G[10$, p. 297].

2.3. Proposition. Let $G \in[F I A]_{B}^{-}$, with $B J I(G)$. Let $K$ be a compact $B$-invariant subgroup, and assume that $G / K$ is $B \cdot f i x e d$; that is $\beta(x) K=x K$ for all $x \in G$ (in particular $G / K$ is abelian). Let $\varphi \in X^{B}(G)$. Then there is an open subgroup $S=S(\varphi)$, containing $K$, with the following properties:

(i) $S=\{x \in G \mid \varphi(y) \neq 0$ for some $y \in x K\}$.

(ii) The function $x \mapsto \int_{K}|\varphi(x k)|^{2} d k$ assumes a constant value $c>0$ on $S$, and vanishes on $G \backslash S$ (dk is normalized Haar measure on $K$ ). 
For each $\lambda \epsilon(S / K)$, regard $\lambda$ as an ordinary one-dimensional character of $S$, constant on cosets of $K$, and let $\lambda^{\sim}$ denote the trivial extension of $\lambda$ to $G$. Let $W(\varphi)$ denote the set of all $\psi \in \mathcal{X}^{B}(G)$ sucb that $\psi_{K} \equiv \varphi_{K^{*}}$

(iii) $W(\varphi)$ is an open and closed subset of $\mathscr{X}^{B}(G)$, and the map $\lambda \mapsto \lambda^{\sim} \varphi$ is a bomeomorphism of $(S / K)^{\wedge}$ onto $W(\varphi)$ (see also [8, Lemma 4]).

(iv) If $\psi \in \mathfrak{X}^{B}(G)$, and $\psi_{K} \equiv \varphi_{K^{\prime}}$ then $\int_{K} \varphi(x k) \overline{\psi(x k)} d k=0$ for all $x \in G$.

Proof. Let $G^{*}$ be the semidirect product $G^{*}=G \times{ }_{\eta} B^{-}$, with $B^{-}$acting in the usual way; let $B^{*}=\left\{(1, \beta): \beta \in B^{-}\right\}$and $K^{*}=\left\{(k, \beta): k \in K, \beta \in B^{-}\right\}$be the corresponding compact subgroups of $G^{*} . K^{*}$ is a normal subgroup of $G^{*}$ : in fact, if $(x, \alpha) \in G^{*}$ and $(k, \beta) \in K^{*}$, then

$$
\begin{aligned}
(x, \alpha)(k, \beta)(x, \alpha)^{-1} & =(x, \alpha)(k, 1)(x, \alpha)^{-1} \cdot(x, \alpha)(1, \beta)(x, \alpha)^{-1} \\
& =\left(x \alpha(k) x^{-1}, 1\right)\left(x \cdot \alpha \beta \alpha^{-1}\left(x^{-1}\right), \alpha \beta \alpha^{-1}\right) .
\end{aligned}
$$

Since $K$ is $B$-invariant (hence normal) in $G$, the first term is in $K^{*}$, and since $G / K$ is $B$-fixed the second term is also in $K^{*}$. Moreover, it is easy to see that the inclusion map $j: x \rightarrow(x, 1)$ induces an isomorphism of $G / K$ onto $G^{*} / K^{*}$. Let $\varphi^{*}(x, \beta)=\varphi(x)$ for $(x, \beta) \in G^{*}$; then as we have noted above, $\varphi^{*}$ is a positive-definite spherical function on $G^{*}$, with respect to $B^{*}$. Let $S\left(\varphi^{*}\right)$ be the subgroup of $G^{*}$ whose existence is proved in 2.2 , and let $S(\varphi)$ denote the inverse image in $G$ of $S\left(\varphi^{*}\right)$, under the map $j$. Then $S(\varphi)$ is an open subgroup of $G$, and it is easy to check that (i), (ii), and (iv) follow from the corresponding conditions in 2.2.

For (iii), we observe first that whenever $\varphi \in \mathfrak{X}^{B}(G), \varphi_{K}$ is actually a $B_{K^{-}}$ character of $K$ (where $B_{K}$ is the group of restrictions to $K$ of the elements of $B$ ); this follows immediately from Proposition 1.1 (iii). The map $\varphi \mapsto \varphi_{K}$ is clearly continuous (in the topology of uniform convergence on compacta) and $\mathfrak{X}^{B} K_{(K)}=X_{(K) / B_{K}^{-}}$is discrete (see [10, Theorem 5.8]). Hence $W(\varphi)$, the inverse image of $\varphi_{K}$ under this mapping, is open and closed. Now if $\varphi$ and $\psi$ are $B$-characters of $G$ which agree on $K$, then $\varphi^{*}$ and $\psi^{*}$ are spherical functions on $G^{*}$ which agree on $K^{*}$. Using 2.2 it follows easily that $\psi=\lambda^{\sim} \varphi$ for a unique $\lambda \epsilon(S / K)^{\wedge}$, so $\lambda \mapsto \lambda^{\sim} \varphi$ is a bijection of $(S / K)^{\wedge}$ onto $W(\varphi)$. On the other hand, a straightforward argument shows that $\lambda_{\nu} \mapsto \lambda$ uniformly on compacta of $S / K$ iff $\lambda_{\nu} \varphi \rightarrow \lambda^{\sim} \varphi$ uniformly on compacta of $G$, so the map is a homeomorphism. Thus the proposition is proved.

2.4. Proposition. Let $G$ be an $[F I A]_{B}^{-}$group, with $B \supset I(G)$. Then $Z^{B}\left(L^{1}(G)\right)$ is a regular commutative Banach algebra.

Proof. The proof is accomplished through a series of reductions, using the structure theory for $[F I A]_{B}^{-}$groups of Grosser and Moskowitz [4]. We show 
first that it suffices to prove the theorem when $G$ is compactly generated. In fact, let $\varphi_{0} \in X^{B}(G)$, and let $W$ be an open neighborhood of $\varphi_{0}$ in $X^{B}(G)$. We must find $f \in Z^{B}\left(L^{1}(G)\right)$ such that $\hat{f}\left(\varphi_{0}\right) \neq 0, \hat{f} \equiv 0$ outside $W$. We may suppose that $W$ is a neighborhood of the form $\left\{\varphi \in X^{B}(G):\left|\varphi(x)-\varphi_{0}(x)\right|<\epsilon\right.$ for all $\left.x \in K\right\}$, where $\epsilon>0$ and $K$ is a compact set with nonempty interior; we may also assume that $K$ is $B$-invariant, by considering if necessary the compact set $B^{-}(K)$. Let $H$ be the open compactly generated $B$-invariant subgroup of $G$ generated by $K$, and let $C=B_{H}$ be the group of restrictions to $H$ of elements of $B$. Let $\chi_{0}=$ $\left(\varphi_{0}\right)_{H}$, and for $\varphi \in X^{B}(G)$, let $\chi=\varphi_{H}$. Then $\chi_{0}$ and $\chi$ are $C$-characters of $H$ [10, Proposition 2.9], and $\varphi \in W$ if and only if $\chi \in W_{H}=\left\{\psi \in X^{C}(H):\left|\psi(x)-\chi_{0}(x)\right|\right.$ $<\epsilon$ for all $x \in K$, a neighborhood of $\chi_{0}$ in $x^{C}(H)$. If the theorem is valid for compactly generated groups, then there exists $g \in Z^{C}\left(L^{1}(H)\right)$ such that $\hat{g}\left(\chi_{0}\right)=1$ and $\hat{g} \equiv 0$ on $X^{C}(H) \backslash W_{H}$. Let $g^{\sim}$ be the trivial extension of $g$ to $G$, obtained by setting $g^{\sim}(x)=0$ for $x \in G \backslash H$. Then $\left(g^{\sim}\right)^{\wedge}(\varphi)=\hat{g}(\chi)$ for all $\varphi \epsilon$ $X^{B}(G)$, and $g^{\sim} \in Z^{B}\left(L^{1}(G)\right)$; hence $g^{\sim}$ satisfies the requirements of the theorem.

We may therefore assume that $G$ is compactly generated. On the other hand, to prove the proposition for a given group $G$ and group of automorphisms $B D(G)$, it is sufficient to prove the theorem for $G$ and $I(G)$. For suppose this latter case has already been proved; again let $\varphi_{0} \in \mathfrak{X}^{B}(G)$ and let $W$ be an open neighborhood of $\varphi_{0}$ in $\mathcal{X}^{B}(G)$. By [10, Theorem 5.8], we know that the map $\chi \mapsto \chi^{\#}$, where $\chi^{\#}(x)=\int_{B}-\chi(\beta x) d \beta$, maps $X(G)$ continuously onto $X^{B}(G)$. Let $W^{\prime}=\left\{\chi \in X(G): \chi^{\#} \in W\right\}$ and pick $\chi_{0} \in W^{\prime}$ such that $\chi_{0}^{\#}=\varphi_{0}$. By our assumption, there exists $g \in Z\left(L^{1}(G)\right)$ such that $\hat{g}\left(\chi_{0}\right)=1$ and $\hat{g}$ vanishes outside $W^{\prime}$. Let $f=\left(g{ }^{*} * g\right)^{\#}$; then $f \in Z^{B}\left(L^{1}(G)\right)$ and by Fubini's theorem

$$
\begin{aligned}
\hat{f}\left(\chi^{\#}\right) & =\int_{G}\left(g^{\sim} * g\right) \#(x) \chi^{\#}(x) d x=\int_{G}\left(g^{\sim} * g\right)(x) \chi^{\#}(x) d x \\
& =\int_{B}-|\hat{g}(\chi \circ \beta)|^{2} d \beta .
\end{aligned}
$$

Thus $\hat{f}\left(\varphi_{0}\right)>0$, and if $\varphi$ is outside $W, \hat{f}(\varphi)=0$.

We are therefore reduced to showing that if $G$ is a compactly generated [FIA] $]^{-}$group, then $Z\left(L^{1}(G)\right)$ is regular. It follows from [4, Theorem 3.20] that $G \in[F D]^{-}$; that is, $G$ contains a compact nomal subgroup $K$ such that $G / K$ is abelian. Again let $\varphi_{0} \in \mathcal{X}(G)$, and let $W$ be an open neighborhood of $\varphi_{0}$ in $X(G)$. By intersecting with $W\left(\varphi_{0}\right)$ (see 2.3 ) if necessary, we may assume $W \subset W\left(\varphi_{0}\right)$. If $S=S\left(\varphi_{0}\right)$ is the subgroup discussed in 2.3 , then there is an open neighborhood $U$ of 1 in $(S / K)^{\wedge}$ which is mapped onto $W$ via the homeomorphism $\lambda \mapsto \lambda^{\sim} \varphi_{0}$. We now pick $g \in L^{1}(S / K)$ such that $\hat{g}(1)=1, \hat{g}(\lambda)=0$ for $\lambda \in(S / K)^{\wedge} \backslash U$. Regarding $g$ as a function on $S$, we let $f=g^{\sim} \bar{\varphi}_{0}\left(\bar{\varphi}_{0}\right.$ is the complex conjugate of $\left.\varphi_{0}\right)$. We shall show that $f$ satisfies the requirements of the proposition. In fact, $g^{\sim} \epsilon$ $L^{1}(G)$ and $\bar{\varphi}_{0}$ is bounded, so $f \in L^{1}(G)$; moreover $f$ is central, since $g^{\sim}$ is con- 
stant on $K$ cosets and $G / K$ is abelian. Also,

$$
\begin{aligned}
\hat{f}\left(\varphi_{0}\right) & =\int_{G} g^{\sim}(x) \bar{\varphi}_{0}(x) \varphi_{0}(x) d x=\int_{S} g(x)\left|\varphi_{0}(x)\right|^{2} d x \\
& =\int_{S / K} g(\dot{x}) \int_{K}\left|\varphi_{0}(x k)\right|^{2} d k d \dot{x}=c \int_{S / K} g(\dot{x}) d \dot{x}=c \hat{g}(1) \neq 0,
\end{aligned}
$$

where $c$ is the constant in 2.3 (ii). Finally, if $\varphi \in \mathcal{X}(G) \backslash W$, then either $\varphi \in$ $W\left(\varphi_{0}\right) \backslash W$, or $\varphi \in \mathcal{X}(G) \backslash W\left(\varphi_{0}\right)$. In the first case, $\varphi=\lambda^{\sim} \varphi_{0}$ for some $\lambda \in(S / K)^{\wedge} \backslash U$; hence

$$
\begin{aligned}
\hat{f}(\varphi) & =\int_{S} g(x) \lambda(x)\left|\varphi_{0}(x)\right|^{2} d x=\int_{S / K} g(\dot{x}) \lambda(\dot{x}) \int_{K}\left|\varphi_{0}(x k)\right|^{2} d k d \dot{x} \\
& =c \hat{g}(\lambda)=0
\end{aligned}
$$

by hypothesis. In the second case,

$$
\hat{f}(\varphi)=\int_{S} g(x)_{\varphi_{0}}(x) \varphi(x) d x=\int_{S / K} g(\dot{x}) \int_{K} \overline{\varphi_{0}(x k)} \varphi(x k) d k d \dot{x}=0
$$

by 2.3 (iv). This proves the proposition.

The following corollary was proved for groups of the form $A \times K$, with $A$ abelian and $K$ compact, by A. Willcox [18].

2.5. Corollary. Let $G$ be an $[F I A]^{-}$group. Then $L^{1}(G)$ is completely regular (in the sense of [15]).

Proof. $L^{1}(G)$ is a weakly central Banach algebra (that is, the map $M \mapsto$ $M \cap Z\left(L^{1}(G)\right)$ is injective from the set of regular maximal two-sided ideals of $L^{1}$, to the set of regular maximal ideals of $Z\left(L^{1}(G)\right)$ ), as indicated in Proposition 4.5 of [10]. Since $Z\left(L^{1}(G)\right)$ is regular, the result now follows immediately from $[15,2.7 .9]$.

As in 2.4, the proof of the following proposition is accomplished through a series of reductions. We recall that the maximal ideal space of $Z^{B}\left(L^{1}(G)\right)$ is just $\mathscr{X}^{B}(G)$ (see 1.1) in case $G \in[F I A]_{B}^{-}$with $B \supset I(G)$.

2.6. Proposition. Let $G$ be an $[F I A]_{B}^{-}$group, with $B \supset I(G)$. Then $\mathrm{Z}^{B}\left(L^{1}(G)\right)$ is Tauberian.

Proof. We show first that it suffices to consider the case when $G$ is compactly generated. In fact, let $f \in Z^{B}\left(L^{1}(G)\right)$, and choose $g$, a continuous $B$. invariant function with compact support, such that $\|f-g\|_{L^{1}(G)}$ is small (this can be done since the map $f \mapsto f^{\#}, f^{\#}(x)=\int_{B^{-}} f(\beta x) d \beta$, is norm decreasing, takes functions into $B$-invariant functions, and takes continuous functions with compact support into continuous functions with compact support $[10, \$ 1]$ ). Let $H$ be the open, compactly generated, $B$-invariant subgroup of $G$ generated by supp $g$. Let $C=B_{H}$, and (assuming the proposition true for $H$ ) find $b \epsilon$ $Z^{C}\left(L^{1}(H)\right)$ such that $\|g-b\|_{L^{1}(H)}$ is small, and $\hat{b}$ has compact support in 
$X^{C}(H)$. Let $b^{\sim}$ be the trivial extension of $b$ to $G: b^{\sim}(x)=0$ for $x \in G \backslash H$. We claim that $b^{\sim} \in Z^{B}\left(L^{1}(G)\right),\left\|g-b^{\sim}\right\|_{L^{1}(G)}$ is small, and $\left(b^{\sim}\right)^{\wedge}$ has compact support in $\mathcal{X}^{B}(G)$. The first two assertions are obvious. For the third, let $K C$ $\mathscr{X}^{C}(H)$ be the support of $\hat{h}$, and let $\mathcal{K}^{\prime}=\left\{\varphi \in \mathcal{X}^{B}(G): \varphi_{H} \in \mathcal{K}\right\}$. If $r: \mathscr{X}^{B}(G) \rightarrow$ $X^{C}(H)$ is the restriction map $r(\varphi)=\varphi_{H}[10,2.9]$, then $\mathcal{K}^{\prime}=r^{-1}(\mathcal{K})$. Moreover, $\mathcal{K}^{\prime}$ is compact. In fact, if we put $r(0)=0$, then $r: \mathfrak{X}^{B}(G) \cup\{0\} \rightarrow X^{C}(H) \cup\{0\}$ is clearly a continuous map in the respective weak- ${ }^{*}$ topologies. Since $X B(G) \cup$ $\{0\}$ is the one-point compactification of $\mathcal{X}^{B}(G)$, and similarly for $\mathcal{X}^{C}(H), r^{-1}$ takes neighborhoods of $\infty$ to neighborhoods of $\infty$ and therefore compact sets to compact sets. Since $\left(b^{\sim}\right)^{\wedge}(\varphi)=\hat{b}\left(\varphi_{H}\right),\left(b^{\sim}\right)^{\wedge}$ has its support in $\mathcal{K}^{\prime}$.

We may therefore assume that $G$ is compactly generated. On the other hand, to prove the theorem for a relatively compact subgroup $B C \operatorname{Aut}(G), B \supset I(G)$, it suffices to prove the theorem for $I(G)$. In fact, suppose we have proved that the set of all $f \in Z\left(L^{1}(G)\right.$ ), such that $\hat{f}$ has compact support in $\mathcal{X}(G)$, is dense in $Z\left(L^{1}(G)\right)$. Then given $g \in Z^{B}\left(L^{1}(G)\right) \subset Z\left(L^{1}(G)\right)$, there exists such an $f$ with $\|g-f\|$ small. But this implies that $\left\|g-f^{\#}\right\|$ is small (since $f \mapsto f^{\#}$ is norm decreasing and $g$ is $B$-invariant), and it is easy to see that $\left\{\varphi \in \mathscr{X}^{B}(G):\left(f^{\#}\right)^{\wedge}(\varphi)\right.$ $\not 0\}$ is contained in $\left\{\chi^{\#}: \chi \in \operatorname{supp} \hat{f}\right.$ in $\left.\mathcal{X}(G)\right\}$. Since $\dot{\chi} \mapsto \chi^{\#}$ is a continuous map of $X_{(G)}$ onto $X^{B}(G)[10,5.8],\left(f^{\#}\right)^{\wedge}$ has compact support.

It therefore suffices to prove the theorem when $G$ is a compactly generated $[F I A]^{-}$group. In this case $G$ can be written as a direct product $V \times H$, where $V$ and $H$ have the following properties: $V$ is a vector group; $H$ is an $[F I A]^{-}$ group containing a compact open normal subgroup $K$ such that $H / K$ is abelian. We observe first that the functions of the form $f \otimes g$ (where $f \otimes g(x, y)=$ $f(x) g(y))$ with $f \in L^{1}(V)$ and $g \in Z\left(L^{1}(H)\right)$, form a total subset of $Z\left(L^{1}(G)\right)$. In fact, any $b \in L^{1}(G)$ can be approximated in $L^{1}(H)$ by finite sums of the form $\Sigma f_{i} \otimes g_{i}$, with $f_{i} \in L^{1}(V)$ and $g_{i} \in L^{1}(H)$. If $b \in Z\left(L^{1}(G)\right)$, then $b=b^{\#}$ is approximated by $\Sigma\left(f_{i} \otimes g_{i}\right)^{\#}=\Sigma f_{i} \otimes\left(g_{i}\right)^{\#}$ (here ${ }^{\#}$ is with respect to $I(G)$ ). Hence we need only show that $f \otimes g$ can be approximated by a function in

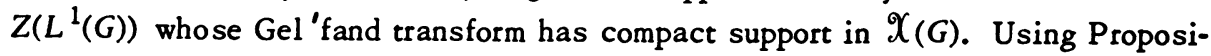
tion 1.1 (iii) it is easy to show that the map $(\psi, \varphi) \mapsto \psi \otimes \varphi$ is a homeomorphism of $\hat{V} \times X_{(H)}$ onto $\mathcal{X}(G)$. Thus if we can prove the theorem for $V$ and $H$ separately, its validity for $G$ will follow. Now the assertion is well known for $V$, so without loss of generality we assume $G=H$ contains a compact open normal subgroup $K$ with $G / K$ abelian. Let $\mathcal{F}=\left\{f \in L^{1}(G)\right.$ : $f$ agrees a.e. with a function constant on $K$ cosets $\}$. We show that functions of the form $f \varphi$, where $f \in \mathcal{F}$ and $\varphi \in \mathcal{X}(G)$ : (i) have Gel 'fand transforms with compact support in $\mathcal{X}(G)$, and (ii) form a total subset of $Z\left(L^{1}(G)\right)$. Clearly $f \varphi$ is central since $G / K$ is

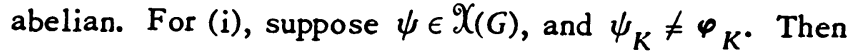




$$
(f \varphi)^{\wedge}(\psi)=\int_{G} f(x) \varphi(x) \psi(x) d x=\sum_{\dot{x} \in G / K} f(\dot{x}) \int_{K} \varphi(x k) \psi(x k) d k=0
$$

by Proposition 2.3 (iv). Hence $\operatorname{supp}(f \varphi)^{\wedge}$ is contained in the set $W(\bar{\varphi})=(S(\bar{\varphi}) / K)^{\wedge} \cdot \bar{\varphi}$. Since $K$ is open, $(S(\varphi) / K)^{\wedge}$ is compact, and $(f \varphi)^{\wedge}$ has compact support. On the other hand, it is easy to show that if $\langle$,$\rangle denotes the usual duality be-$ tween $L^{1}(G)$ and $L^{\infty}(G)$, then $f \mapsto\langle, f\rangle$ is an isometric isomorphism of $Z\left(L^{\infty}(G)\right.$ ) onto $Z\left(L^{1}(G)\right)^{\prime}$ (where $Z\left(L^{\infty}(G)\right.$ ) denotes the space of $L^{\infty}$ functions which are invariant under inner automorphisms). Thus in order to prove (ii), let $\psi \in Z\left(L^{\infty}(G)\right)$ and suppose $\langle/ \varphi, \psi\rangle=0$ for all $f \in \mathcal{F}$ and $\varphi \in \mathcal{X}(G)$. Then $f \psi \in Z\left(L^{1}(G)\right)$ and we have $(f \psi)^{\wedge}(\varphi)=\langle f \psi, \varphi\rangle=\langle f \varphi, \psi\rangle=0$ for all $\varphi \in \mathcal{X}(G), f \in \mathcal{F}$. Since $Z\left(L^{1}(G)\right)$ is semisimple, $f \psi=0$ for all $f \in \mathcal{F}$. In particular, taking $f$ to be the characteristic function of an arbitrary coset $x K$, we conclude $\psi=0$. Therefore \{f $\varphi: f \in \mathcal{F}$ and $\varphi \in \mathcal{X}(G)\}$ is a total subset of $Z\left(L^{1}(G)\right)$.

2.7. Proof of Theorem 1.8. In view of 1.5 , we need only show that $Z^{B}\left(L^{1}(G)\right.$ ) is a regular, Tauberian Banach algebra, provided $G \in[F I A]_{B}^{-}$with $B \supset I(G)$. This is the content of Propositions 2.4 and 2.6.

2.8. Remark. If $G$ is an $[F I A]^{-}$group, then Wiener's Tauberian theorem holds in the following form for $L^{1}(G)$ : If $M$ is a proper closed two-sided ideal in $L^{1}(G)$, then $M$ is contained in a regular maximal two-sided ideal of $L^{1}(G)$. In fact, $M \cap Z\left(L^{1}(G)\right)$ is a proper closed ideal in $Z\left(L^{1}(G)\right.$ ) (otherwise $M$ would contain an approximate identity for $L^{1}(G)$ ), hence by 1.9 is contained in a regular maximal ideal of $Z\left(L^{1}(G)\right)$. It now follows from the proof of $[10,4.5]$ that $M$ is contained in a regular maximal two-sided ideal of $L^{1}(G)$.

3. In this section we investigate the validity of the condition of Wiener-Ditkin for the algebra $Z\left(L^{1}(G)\right)$. In general, a commutative Banach algebra $A$ with maximal ideal space $\Delta(A)$ is said to satisfy the condition of Wiener-Ditkin (WD) [14, p. 27] if given $\varphi \in \Delta(A), f \in A$, with $\hat{f}(\varphi)=0$, and $\epsilon>0$, there exists $g \in A$ such that $\hat{g} \equiv 1$ in a neighborhood of $\varphi$ and $\|f g\|<\epsilon$. We show now that if $G \epsilon$ $[F D]^{-}$, then $Z\left(L^{1}(G)\right)$ satisfies the condition (WD). We conclude the paper with an example of a locally compact group $G$ for which $Z\left(L^{1}(G)\right.$ ) does not satisfy the condition (WD).

3.1. Proposition. Let $G$ be an $[F D]^{-}$group. Then $Z\left(L^{1}(G)\right)$ satisfies the condition of Wiener-Ditkin.

Proof. Let $K$ be a compact normal subgroup of $G$ such that $G / K$ is abelian. Suppose $\varphi \in \mathcal{X}(G), f \in Z\left(L^{1}(G)\right)$ with $\hat{f}(\varphi)=0$, and $\epsilon>0$ are given. Let $S=$ $S(\varphi)=\{x \in G: \varphi(y) \neq 0$ for some $y \in x K\}$ be the open subgroup of 2.3; then the central $L^{1}$ function $f \varphi$ is supported in $S$. Let $b$ be the restriction of $f \varphi$ to $S$, and let $b^{\prime}$ be the $L^{1}$ function on $S / K$ defined by $b^{\prime}(\dot{x})=\int_{K} b(x k) d k$. Then 


$$
\hat{b^{\prime}}(1)=\int_{S / K} b^{\prime}(\dot{x}) d \dot{x}=\int_{S} b(x) d x=\hat{f}(\varphi)=0 .
$$

Since $S / K$ is abelian, the condition (WD) holds for $L^{1}(S / K)[14$, p. 125]; hence there exists $\tau^{\prime} \in L^{1}(S / K)$ such that $\left(r^{\prime}\right)^{\wedge} \equiv 1$ in a neighborhood $U$ of 1 in $(S / K)^{\wedge}$, and $\left\|b^{\prime} * r^{\prime}\right\|_{L^{1}(S / K)}<\epsilon$. Let $\theta: S \rightarrow S / K$ be the canonical surjection, and let $r=r^{\prime} \circ \theta$. Then $b^{\prime} * r^{\prime}=(b * r)^{\prime}$, and $b * r$ is constant on cosets of $K$, so $\|b * r\|_{L^{1}(S)}=\left\|b^{\prime} * r^{\prime}\right\|_{L^{1}(S / K)}<\epsilon[6,28.55]$. Finally, let $r^{\sim}$ be the trivial extension of $\tau$ to a function on $G$, and let $g=(1 / c) r^{\sim} \bar{\varphi}$, where $c$ is the constant value of $\int_{K}|\varphi(x k)|^{2} d k$ on $S(2.3$ (ii)). We will show that $g$ satisfies the requirements of condition (WD) at $\varphi$. It is clear, first, that $g \in Z\left(L^{1}(G)\right)$, since $G / K$ is abelian. Second, if we regard each element $\lambda$ of $(S / K)^{\wedge}$ as a function on $S$, constant on cosets of $K$, then the map $\lambda \mapsto \lambda^{\sim} \varphi$ is a homeomorphism of $U$ onto a neighborhood $W$ of $\varphi\left(2.3\right.$ (iii)). For any $\lambda^{\sim} \varphi \in W$,

$$
\begin{aligned}
\hat{g}(\lambda \sim \varphi) & =\frac{1}{c} \int_{S} \lambda(x) \tau(x)|\varphi(x)|^{2} d x=\frac{1}{c} \int_{S / K} \lambda(\dot{x}) \tau^{\prime}(\dot{x}) \int_{K}|\varphi(x k)|^{2} d k d \dot{x} \\
& =\left(r^{\prime}\right)^{\wedge}(\lambda)=1 .
\end{aligned}
$$

Thus $\hat{g} \equiv 1$ on $W$. Finally, $f * g$ is central; hence $f * g(x)=\int_{I(G)}-f * g(\beta(x)) d \beta$ for almost all $x_{\text {. There }}$ There using 1.1 (iii) we have

$$
\begin{aligned}
f * g(x) & =\frac{1}{c} \int_{I(G)}-\int_{G} f(y) \tau^{\sim}\left(y^{-1} \beta(x)\right) \bar{\varphi}\left(y^{-1} \beta(x)\right) d y d \beta \\
& =\frac{1}{c} \int_{G} f(y) \sim\left(y^{-1} x\right) \int_{I(G)}-\varphi\left(\beta\left(x^{-1}\right) y\right) d \beta d y \\
& =\frac{1}{c} \int_{G} f(y) \sim\left(y^{-1} x\right) \varphi(y) \overline{\varphi(x)} d y \\
& =0, \quad x \in G^{\prime} S, \\
& =\frac{1}{c} \overline{\varphi(x)} b * \tau(x), \quad x \in S .
\end{aligned}
$$

Thus $\|f * g\|_{1} \leq \epsilon / c$. Since $c$ is independent of $\epsilon$, the proof is complete.

We refer the reader to $[14, \mathrm{pp} .20,28]$ for the terminology used in the next corollary.

3.2. Corollary. Let $G \in[F D]^{-}, M$ a closed ideal in $Z\left(L^{1}(G)\right)$, and $f \epsilon$ $Z\left(L^{1}(G)\right)$ such that $\hat{f}$ vanishes on $\operatorname{cosp} M$. If the boundaries $\partial \operatorname{cosp} f$ and $\partial \operatorname{cosp} M$ bave the property that $\partial \operatorname{cosp} / \cap \partial \operatorname{cosp} M$ contains no perfect subset, then $f \in M$. In particular, any closed set in $X(G)$ whose boundary contains no perfect subsets is a Wiener set (tbat is, a set of spectral synthesis). •

Proof. $Z\left(L^{1}(G)\right)$ is a regular Tauberian Banach algebra (1.8) with approximate identity (1.6) satisfying the condition (WD). The corollary then follows immediately from Theorem 4.6 of Chapter 2 of [14]. 
We conclude the paper with an example of a locally compact group $G$ for which $Z\left(L^{1}(G)\right)$ does not satisfy (WD). If we let $G=\mathbf{R}^{3} \times{ }_{\eta} S O(3)$, where $S O(3)$ is given the discrete topology and acts on $\mathrm{R}^{3}$ in the natural way, then $Z\left(L^{1}(G)\right)$ consists of the radial $L^{1}$ functions on $R^{3}$ under convolution. By 6.3 of Chapter 2 of [14], this last algebra does not satisfy (WD). We do not know, however, whether the condition (WD) is always satisfied for $Z\left(L^{1}(G)\right)$ when $G \in[F C]^{-}$.

\section{BIBLIOGRAPHY}

1. J. Dixmier, Les $C^{*}$-algèbres et leurs représentations, Cahiers Scientifiques, fasc. 29, Gauthier-Villars, Paris, 1964. MR 30 \#1404.

2. R. Godement, Introduction aux travaux de A. Selberg, Séminaire Bourbaki 1956/57, Secrétariat mathématique, $P$ aris, 1959.

3. S. Grosser, R. Mosak, and M. Moskowitz, Duality and harmonic analysis on central topological groups, Indag. Math. 35 (1973), 65 - 91.

4. S. Grosser and M. Moskowitz, Compactness conditions in topological groups, J. Reine Angew. Math. 246 (1971), 1-40. MR 44 \#1766.

5. S. Helgason, Differential geometry and symmetric spaces, Pure and Appl. Math., vol. 12, Academic Press, New York, 1962. MR 26 \#2986.

6. E. Hewitt and K. A. Ross, Abstract harmonic analysis. Vol. II, Die Grundlehren der math. Wissenschaften, Band 152, Springer-Verlag, Berlin and New York, 1970. MR $41 \# 7378$.

7. A. Hulanicki, $O_{\text {n }}$ positive functions on a group algebra multiplicative on a subalgebra, Studia Math. 37 (1971), 163-171.

8. E. Kaniuth and G. Schlichting, Zur harmonischen Analyse klassenkompakter Gruppen. II, Invent. Math. 10 (1970), 332-345.

9. J. Liukkonen, Dual spaces of locally compact groups with precompact conjugacy classes, Tran s. Amer. Math. Soc. 180 (1973), 85-108.

10. R. Mosak, The $L^{1}$ - and $C^{*}$-algebras of $[F I A]_{B}^{-}$groups, and their representations, Trans. Amer. Math. Soc. 163 (1972), 277 - 310. MR 45 \#2096.

11. Central functions in group algebras, Proc. Amer. Math. Soc. 29 (1971), 613-616. MR 43 \#5323.

12. R. Mosak and M. Moskowitz, Central idempotents in measure algebras, Math. Z. 122 (1971), 217 - 222. MR 45 \#9062.

13. P. Porcelli and H. Collins, Ideals in group algebras, Studia Math. 33 (1969), 223 226. MR 40 \#4779.

14. H. Reiter, Classical harmonic analysis and locally compact groups, Oxford mathematical monographs, Oxford University Press, Oxford, 1968.

15. C. Rickart, General theory of Banach algebras, University Series in Higher Mathematics, Van Nostrand, Princeton, N.J., 1960. MR 22 \#5903.

16. L. Robertson, $A$ note on the structure of Moore groups, Bull. Amer. Math. Soc. 75 (1969), 594-599. MR 39 \#7027.

17. I. Segal, The group algebra of a locally compact group, Trans. Amer. Math. Soc. 61 (1947), 69-105. MR 8, 438. 
18. A. Willcox, Note on certain group algebras, Proc. Amer. Math. Soc. 7 (1956), 874-879. MR 19, 46.

DEPARTMENT OF MATHEMATICS, TULANE UNIVERSITY, NEW ORLEANS, LOUISIANA 70118

DEPARTMENT OF MATHEMATICS, YALE UNIVERSITY, NEW HAVEN, CONNECTICUT 06520 (Current address of J. Liukkonen)

Current address (R. Mosak): Department of Mathematics, University of Rochester, Rochester, New York 14627 